\title{
A NUMERICAL BIFURCATION FUNCTION FOR HOMOCLINIC ORBITS*
}

\author{
PETER ASHWIN ${ }^{\dagger}$ AND ZHEN MEI ${ }^{\ddagger}$
}

\begin{abstract}
We present a numerical method to locate periodic orbits near homoclinic orbits. Using a method of [X.-B. Lin, Proc. Roy. Soc. Edinburgh, 116A (1990), pp. 295-325] and solutions of the adjoint variational equation, we get a bifurcation function for periodic orbits, whose periods are asymptotic to infinity on approaching a homoclinic orbit. As a bonus, a linear predictor for continuation of the homoclinic orbit is easily available.

Numerical approximation of the homoclinic orbit and the solution of the adjoint variational equation are discussed. We consider a class of methods for approximating the latter equation such that a scalar quantity is preserved. We also consider a context where the effects of continuous symmetries of equations can be incorporated.

Applying the method to an ordinary differential equation on $R^{3}$ studied by [E. Freire, A. RodriguezLuis, and E. Ponce, Phys. D, 62 (1993), pp. 230-253] we show the bifurcation function gives good agreement with path-followed solutions even down to low period. As an example application to a parabolic partial differential equation (PDE), we examine the bifurcation function for a homoclinic orbit in the Kuramoto-Sivashinsky equation.
\end{abstract}

Key words. periodic solutions, homoclinic orbit, numerical bifurcation function

AMS subject classifications. 34C $35,34 \mathrm{C} 37,34 \mathrm{C} 25,58 \mathrm{~F} 14,65 \mathrm{~L} 10$

PII. S0036142996298168

1. Introduction. This paper is concerned with dynamical behavior near a homoclinic orbit of the parameter dependent autonomous differential equation

$$
\dot{x}(t)=f(x(t), \mu),
$$

where $f: R^{n} \times R^{d} \rightarrow R^{n}$ is a $C^{k}$-continuous $(k \geq 2)$ mapping and $\mu \in R^{d}$ represents the control parameters. This ODE may have been derived as a large system from a spatial discretization of a parabolic PDE.

Suppose that at $\mu=0$ the flow has a homoclinic orbit $q(t)$ which is a solution of the system (1) and which converges to an equilibrium $p$ as $t \rightarrow \pm \infty$, i.e.,

$$
p=\lim _{t \rightarrow \pm \infty} q(t)
$$

Furthermore, we assume that the equilibrium $p$ is hyperbolic, i.e., the Jacobian matrix $D_{x} f(p, 0)$ has no eigenvalues on the imaginary axis. It is well known that a homoclinic orbit can lead to rich dynamics in the system (1). We are interested in the bifurcation of periodic orbits and the continuation of the homoclinic orbit with respect to control parameters in $\mu$.

*Received by the editors January 31, 1996; accepted for publication (in revised form) November 10, 1997; published electronically August 31, 1998.

http://www.siam.org/journals/sinum/35-5/29816.html

$\dagger$ Institut Non-Linéaire de Nice, 1361 route des Lucioles, 06560 Valbonne, France. This author was supported by the European Union HCM program ERBCHBGCT930503. Present address: Department of Mathematics and Statistics, University of Surrey, Guildford GU2 5XH, UK (p.ashwin@surrey.ac.uk).

${ }^{\ddagger}$ Fachbereich Mathematik, Universität Marburg, Lahnberge, D-35032 Marburg, Germany (meizhen@mathematik.uni-marburg.de). This author was supported by the Deutsche Forschungsgemeinschaft. 
In order to characterize these (and many other) phenomena for a generic set of such systems (notably including those which have one-dimensional intersection of tangent spaces of the stable and unstable manifolds of $p$ along the homoclinic orbit $q(t))$ Lin introduced in [15] a bifurcation function $G(\omega, \mu)$ giving the jump in piecewise smooth solutions of (1) on varying a time interval $\omega \in R^{+}$and parameters $\mu$. Lin showed that for appropriate vectors $\Delta$ and $\bar{\psi}$, for large enough $\omega$ and small enough $\mu$, there is a unique solution $\left(x_{\omega}(t), G(\omega, \mu)\right)$ to the boundary value problem:

$$
\begin{aligned}
\dot{x}-f(x, \mu) & =0 \quad t \in[0,2 \omega], \\
\langle\bar{\psi}, x(0)-q(0)\rangle & =0, \\
x(2 \omega)-x(0) & =G \Delta .
\end{aligned}
$$

Moreover, asymptotically as $\omega \rightarrow \infty$ and $|\mu| \rightarrow 0$, the jump $G(\omega, \mu)$, known as the bifurcation function, can be approximated as

$$
G(\omega, \mu)=\psi(\omega) q(-\omega)-\psi(-\omega) q(\omega)+\sum_{i=1}^{d} \mu_{i} \int_{-\infty}^{\infty} \psi(s) \frac{\partial f}{\partial \mu_{i}}(q(s), 0) d s+\text { h.o.t. }
$$

where h.o.t. refers to higher-order terms and $\psi(t)$ is a nontrivial bounded solution of the so-called adjoint variational equation (AVE)

$$
\dot{\psi}=-\left(D_{x} f(q(t), 0)\right)^{T} \psi .
$$

Zeros of the bifurcation function $G(\omega, \mu)=0$ at finite $\omega$ and small $|\mu|$ are in one-to-one correspondence with period- $2 \omega$ solutions of (1) near the homoclinic orbit $q$. Moreover, solutions of $G(\infty, \mu)=\lim _{\omega \rightarrow \infty} G(\omega, \mu)=0$ give values of $\mu$ for which the system (1) has homoclinic orbits (see also [19]).

In this paper we discuss how (4) can be used as a numerical tool to investigate branching of periodic orbits from and continuation of a homoclinic orbit. We briefly discuss methods for computing homoclinic orbits in section 2 and then move on to the problem of solving the adjoint variational equation. The structure of the AVE means that there is a conserved quantity that must be preserved in a numerical approximation (see (11) below). In section 2.4 we consider implications of continuous symmetries and discuss how one can handle bifurcations of relative periodic orbits from relative homoclinic orbits using a modification of the same method.

Section 3 is devoted to numerical approximations of the bifurcation function (4). We show that a predictor for continuation of the homoclinic orbit is easily available.

Finally in section 4 we examine the equations for an electronic circuit of Freire, Rodriguez-Luis, and Ponce [9] and symmetry breaking perturbations of the KuramotoSivashinsky equation [6] as examples of applications of the presented methods. In the first case we compare the bifurcation function to the periodic orbits obtained from path following with AUTO86. Good agreement is found in both cases even down to low periods.

2. Approximation of the homoclinic orbit and solution of the adjoint problem.

2.1. Approximation of the homoclinic orbit. Several methods are available for the numerical approximation of homoclinic orbits, e.g., shooting methods (cf. Doedel [7]), collocation methods (cf. Moore [16]), and boundary value problems on truncated intervals with projection boundary conditions, (cf. Beyn $[2,3]$ ). We choose 
the latter approach, which has proven to be reliable and stable for many problems; see, e.g., Hassard [10], de Hoog and Weiss [11], Lentini and Keller [14], Beyn [2, 3], and Champneys, Kuznetsov, and Sandstede [5]. One approximates the homoclinic orbit over a finite time interval $[-T, T]$, includes a phase condition, and assumes that at $T$ (resp., $-T$ ) the ends of the orbits are in the tangent spaces of the stable (resp., unstable) manifold to the fixed point. Let $p(\mu)$ be an equilibrium with stable manifold $\mathcal{M}_{s}(\mu)$ and unstable manifold $\mathcal{M}_{u}(\mu)$. Assume $p(\mu)$ has $d_{+}$unstable modes, $d_{-}$stable modes and $d_{0}$ neutral modes $\left(d_{+}+d_{-}+d_{0}=n\right)$. We define $L_{u}(x, \mu)$ to be projection of $x$ onto the eigenspace of unstable modes of $p(\mu), L_{n}(x, \mu)$ onto the neutral modes, and $L_{s}(x, \mu)$ onto stable modes, i.e., $L_{s}(x, \mu)$ can be thought of as linear combinations of $d_{-}$vectors

$$
\left\{\left\langle e_{i}^{*}, x\right\rangle e_{i}: i=1, \ldots, d_{-}\right\},
$$

where $\left\{e_{i}\right\}$ (resp., $\left\{e_{i}^{*}\right\}$ ) are the $d_{-}$right (resp., left) normalized eigenvectors of $D_{x} f(p(\mu), \mu)$ corresponding to the stable eigenvalues; the projections $L_{n}(x, \mu)$ and $L_{u}(x, \mu)$ are defined in a similar manner.

For one-parameter problems, an orbit $q(t)$ for $\mu=0$ that is homoclinic to $p$ is said to be nondegenerate (cf. Beyn [2]), if $p$ is hyperbolic (i.e., $d_{0}=0$ ), the intersection of the manifolds $\mathcal{M}_{s}$ and $\mathcal{M}_{u}$ is one dimensional and the linearized problem

$$
\dot{z}=D_{x} f(q(t), 0) z, \quad \lim _{t \rightarrow \pm \infty} z(t)=0
$$

has only one bounded solution given by $c \dot{q}(t), c \in R$. A sufficient condition for $q(t)$ to be nondegenerate is that solution spaces of the problems (5) and (6) are exactly one dimensional, respectively, and

$$
\int_{-\infty}^{\infty} \psi(s) \frac{\partial f}{\partial \mu}(q(s), 0) d s \neq 0 .
$$

Assume that we can find $p(\mu)$ and the projections $L_{u}(\cdot, \mu), L_{s}(\cdot, \mu)$ for a homoclinic orbit with $\mu$ near $\mu=0$. In order to remove the degeneracy of $q(t$ ) (all time shifts are also homoclinic orbits) we choose a phase condition $\Psi=0$, see, e.g., Beyn [2], with

$$
\begin{aligned}
& \Psi(x)=\langle\dot{\hat{x}}(0),(x(0)-\hat{x}(0))\rangle \quad \text { or } \\
& \Psi(x)=\int_{-\infty}^{\infty}\langle\dot{\hat{x}}(t),(x(t)-\hat{x}(t))\rangle d t
\end{aligned}
$$

for some initial approximation $\hat{x}(t)$ of $q(t)$. (In fact, an arbitrary function that decays fast enough at $\pm \infty$ can be used.) The homoclinic orbit $q(t)$ is approximated by the following boundary value problem on the finite interval $[-T, T]$ :

$$
\begin{aligned}
\dot{x}(t)-f(x(t), \mu) & =0, \quad t \in[-T, T], \\
L_{s}(x(-T)-p(\mu), \mu) & =0, \\
L_{u}(x(T)-p(\mu), \mu) & =0 \\
\Psi(x, \mu) & =0 .
\end{aligned}
$$

As demonstrated by Beyn [3], solutions of this problem converge exponentially to a given nondegenerate homoclinic orbit in phase space and parameter space as $T \rightarrow \infty$. 
On the other hand, since the homoclinic orbit converges exponentially to the equilibrium as $t \rightarrow \infty$, the gridpoints in the interval $[-T, T]$ can be distributed exponentially to achieve uniform accuracy.

There are many ways to solve (8) numerically. For smaller systems one can use Newton iteration to directly solve it with a collocation or finite difference discretization. For larger systems this becomes computationally much more intensive and will be discussed elsewhere. Shooting methods (for cases where $d_{+}$is small) will probably be more tractable for the case of large dimension.

2.2. Solving the adjoint variational problem. The linear variational problem for (1) at a homoclinic orbit $q(t)$ (assumed to be at $\mu=0$ ) is the nonautonomous linear equation

$$
\dot{z}=D_{x} f(q(t), 0) z
$$

This has at least a one-dimensional space of bounded solutions because $z=c \dot{q}$ satisfies the equation for all $c \in R$. We shall consider a case where symmetry may cause additional dimensions of solution space in section 2.4, but in the absence of symmetry it is generic that $\dot{q}(t)$ gives a basis of the solution space.

Associated with this linear equation is the adjoint variational equation

$$
\dot{\psi}=-D_{x} f(q(t), 0)^{T} \psi,
$$

which, if the homoclinic is nondegenerate, will also have precisely one bounded solution. If a solution $\psi(t)$ of (10) is bounded and the fixed point is hyperbolic, it must moreover decay exponentially to zero as $t \rightarrow \pm \infty$.

Conservation properties. There is an important relation that holds between any solutions $z(t)$ of $(9)$ and $\psi(t)$ of (10) even if the orbit is degenerate, namely, that in the space $R^{n}$ the inner product

$$
\langle\psi(t), z(t)\rangle=\text { constant, independent of } t .
$$

This follows by taking time derivatives and substituting in the equations (see Chossat $[6])$ :

$$
\begin{aligned}
\frac{d}{d t}\langle\psi, z\rangle & =\langle\dot{\psi}, z\rangle+\langle\psi, \dot{z}\rangle \\
& =\left\langle-\left(D_{x} f(q(t), 0)\right)^{T} \psi, z\right\rangle+\left\langle\psi, D_{x} f(q(t), 0) z\right\rangle \\
& =0 .
\end{aligned}
$$

In particular, if we wish to compute a bounded solution of (10), the fact that $\dot{q}(t)$ decays to zero at both ends implies that any such solution must obey

$$
\langle\psi(t), \dot{q}(t)\rangle=0
$$

for all $t$.

Based on an approximation $(x(t), \mu)$ of the homoclinic orbit $(q(t), 0)$ in the interval $[-T, T]$, one might hope, therefore, to approximate $\psi(t)$ by numerically solving the linear boundary value problem

$$
\begin{aligned}
\dot{\psi}(t)+\left(D_{x} f(x(t), \mu)\right)^{T} \psi(t) & =0, \quad t \in[-T, T], \\
L_{s}^{*}(\psi(T), \mu) & =0 \\
L_{u}^{*}(\psi(-T), \mu) & =0 \\
\Phi(\psi, \mu) & =0 .
\end{aligned}
$$


Here the projections $L_{s}^{*}(x, \mu), L_{u}^{*}(x, \mu)$ are defined similarly as $L_{s}(x, \mu), L_{u}(x, \mu)$, respectively, by taking right instead of left eigenvectors of $D_{x} f(p(\mu), \mu)$. The normalization condition $\Phi(\psi, \mu)$ is used to exclude the trivial solution and to fix a bounded solution. Because the kernel of the operator $\frac{d}{d t}+\left(D_{x} f(x(t), \mu)\right)^{T}$ is not trivial, one can, for example, choose $\Phi(\psi, \mu)=\langle\psi(0), \Delta\rangle-1$ for some nonzero vector $\Delta$ such that $\langle\psi(0), \Delta\rangle \neq 0$. However, this makes the system (12) overdetermined.

Suppose that we have an approximation $x(t)$ given by a discretization of the finite interval boundary value problem (8) and wish to approximate $\psi(t)$ using (12). Since $x(t)$ is not an exact solution, we cannot expect a priori that the numerical method we use leaves $\langle\psi(t), \dot{x}(t)\rangle$ independent of $t$; if it tends to a nonzero value at either end of the interval $[-T, T]$, then $\psi(t)$ must blow up since $\dot{x}(t)$ decays.

One way to get around this, used by Champneys and Kuznetsov [4], is to replace (10) by a normalized adjoint variational equation for $\hat{\psi}(t):=\psi(t) /\|\psi(t)\|$, the unit normal in the direction of $\psi(t)$. This has a disadvantage (noted by Champneys, Kuznetsov, and Sandstede [5]) that it turns a linear equation into a nonlinear one.

A more robust approach has been developed by Sandstede [17] and used in [5]. They modify the equation to

$$
\dot{\psi}=-D_{x} f(x(t), \mu)^{T} \psi+\epsilon f(x(t), \mu)
$$

where $\epsilon$ is an artificially introduced parameter. If we treat now $\psi$ and $\epsilon$ as the unknowns, we obtain a nonsingular system and $\epsilon$ in the solution should be small. It is a measure for the error from time discretization and truncation of the problems to the finite interval. By using effective methods for boundary value problems, which are possible for small systems, the truncation error will certainly dominate. In fact, $\epsilon$ satisfies

$$
\frac{d}{d t}\langle\dot{x}, \psi\rangle+\epsilon\langle\dot{x}, \dot{x}\rangle=0 \quad \text { for all } t \in[-T, T] .
$$

This method will produce very reasonable approximations $\psi$ such that the bifurcation function (4) can be approximated.

We propose an alternative class of methods that approximate $\psi$ whilst preserving (11) and the linearity exactly.

2.3. Approximation methods that preserve inner product. Let

$$
\left\{\hat{q}_{i}(t) \in R^{n}: i=1, \ldots, n-1\right\}
$$

be a set of linearly independent vectors, piecewise continuous in time that span a space complementary to $\operatorname{span}[\dot{q}(t)]$ in $R^{n}$, for each $t \in R$.

THEOREM 2.1. A bounded solution of (10) satisfies

$$
\begin{aligned}
\left\langle\dot{\psi}(t)+\left(D_{x} f(q(t), 0)\right)^{T} \psi(t), \hat{q}_{i}(t)\right\rangle & =0, \quad i=1, \ldots, n-1, \\
\langle\psi(t), \dot{q}(t)\rangle & =0,
\end{aligned}
$$

where $\langle\cdot, \cdot\rangle$ is the scalar product in $R^{n}$. Conversely, any solution of (13) is also a solution of (10).

Proof. From the above discussion we see that the first part of the conclusion is the representation of (11) with respect to the basis $\left\{\dot{q}(t), \hat{q}_{i}(t) ; i=1, \ldots, n-1\right\}$ of $R^{n}$. Suppose $\psi(t)$ is a solution of (13). The spanning property of $\left\{\hat{q}_{i}(t)\right\}$ implies that

$$
\dot{\psi}(t)+\left(D_{x} f(q(t), 0)\right)^{T} \psi(t)=c \dot{q}(t)
$$


for some scalar $c \in R$. On the other hand, from the last equation of (13) we derive

$$
\begin{aligned}
c\langle\dot{q}(t), \dot{q}(t)\rangle & =c\left\langle\dot{\psi}(t)+\left(D_{x} f(q(t), 0)\right)^{T} \psi(t), \dot{q}(t)\right\rangle \\
& =c[\langle\dot{\psi}(t), \dot{q}(t)\rangle+\langle\dot{\psi}(t), \ddot{q}(t)\rangle] \\
& =c \frac{d}{d t}\langle\psi(t), \dot{q}(t)\rangle \\
& =0 .
\end{aligned}
$$

Hence, $c=0$ and $\psi(t)$ satisfies (10).

For the numerical approximation of $\psi$ and the preservation of the inner product (11), suppose we have an approximation $x(t)$ of $q(t)$. Let $E_{s}^{*}$ and $E_{u}^{*}$ be the stable and unstable eigenspaces of $\left(D_{x} f(p(\mu), \mu)\right)^{T}$. Taking the projections $y_{s}:=L_{s}^{*}(\dot{x}(T), \mu) \in$ $E_{s}$ and $y_{u}^{*}:=L_{u}^{*}(\dot{x}(-T), \mu) \in E_{s}$, we decompose the spaces $E_{s}^{*}$ and $E_{u}^{*}$ as

$$
E_{s}^{*}=\operatorname{span}\left[y_{s}\right] \oplus \tilde{E}_{s}^{*}, \quad E_{u}^{*}=\operatorname{span}\left[y_{u}\right] \oplus \tilde{E}_{u}^{*} .
$$

Define $\tilde{L}_{s}^{*}$ and $\tilde{L}_{u}^{*}$ to be the projections from $R^{n}$ onto the subspaces $\tilde{E}_{s}^{*}$ and $\tilde{E}_{u}^{*}$, respectively. Furthermore, let $\hat{x}_{i}(t), i=1, \ldots, n-1$ be an orthonormal basis for $(\operatorname{span}[\dot{x}(t)])^{\perp}$ in $R^{n}$. We consider the following system of equations for $\psi(t)$ :

$$
\begin{aligned}
\left\langle\dot{\psi}(t)+\left(D_{x} f(x(t), \mu)\right)^{T} \psi(t), \hat{x}_{i}(t)\right\rangle & =0, \quad t \in[-T, T], \quad i=1, \ldots, n-1, \\
\langle\psi(t), \dot{x}(t)\rangle & =0, \quad t \in[-T, T], \\
\tilde{L}_{s}^{*}(\psi(T), \mu) & =0, \\
\tilde{L}_{u}^{*}(\psi(-T), \mu) & =0, \\
\Phi(\psi) & =0 .
\end{aligned}
$$

This is a system of differential-algebraic equations consisting of $n-1$ implicit differential equations and one explicit algebraic equation. The $n-2$ projected boundary conditions and the normalization condition make the system well posed. Since $(p(\mu), \mu)$ is a hyperbolic steady state, we know that $y_{s} \neq 0$ and $y_{u} \neq 0$ for sufficiently large $T$. Thus the projections $\tilde{L}_{s}^{*}$ and $\tilde{L}_{u}^{*}$ can be defined directly with the basis of $E_{s}^{*}$ and $E_{u}^{*}$.

As a simple example for illustration, we consider a finite difference approximation of (14) on the grid $\left\{t_{1}=-T<t_{2}<\cdots<t_{m}=T\right\}$. For the forward difference approximation, we get the following linear system of $m n$ equations in the $m n$ unknowns $\left\{\psi_{i}\left(t_{j}\right): i=1, \ldots, n, j=1, \ldots, m\right\}$

$$
\begin{aligned}
\left\langle\frac{\psi\left(t_{j+1}\right)-\psi\left(t_{j}\right)}{t_{j+1}-t_{j}}+\left(D_{x} f\left(x\left(t_{j}\right), \mu\right)\right)^{T} \psi\left(t_{j}\right), \hat{x}_{i}(t)\right\rangle & =0, j=1, \ldots, m-1, \\
\left\langle\psi\left(t_{j}\right), \dot{x}\left(t_{j}\right)\right\rangle & =0, j=1, \ldots, m, \\
\tilde{L}_{s}^{*}\left(\psi\left(t_{m}\right), \mu\right) & =0, \\
\tilde{L}_{u}^{*}\left(\psi\left(t_{1}\right), \mu\right) & =0, \\
\Phi(\psi) & =0 .
\end{aligned}
$$

Note that $\left\{\hat{x}_{i}(t) \in R^{n} ; i=1, \ldots, n-1\right\}$ can be defined as the standard basis unit vectors excluding one which attains largest modulus inner product with $\dot{x}(t)$. Furthermore, instead of projecting the boundary conditions one could omit the equations $\langle\psi( \pm T), \dot{x}( \pm T)\rangle=0$; in this case the terms $\langle\psi( \pm T), \dot{x}( \pm T)\rangle$ give a measure of the errors in the discretization.

This method inherits the $O(h)$ errors of the forward difference method and so does not provide an accurate method of solution unless the number of gridpoints is 
taken very high. We have investigated this using an efficient solver for sparse linear systems; however, it still involves very high computational effort, especially if the real part of a leading eigenvalues (i.e., those closest to the imaginary axis [4]) is of small magnitude.

One possible higher-order method is to approximate (14) by shooting. In the case that $d_{+}=1$, this means solving a zero-parameter shooting problem. One of our approaches is to use a Gear's method as time integrator between gridpoints, solving (14) first without respecting the conservation equation, and then imposing $\langle\psi(t), \dot{q}(t)\rangle=0$ by projecting orthogonally onto the codimension-one surface with (11). The sum of the squares of these corrections gives a measure of the error in $\psi$. Results of using this method are presented in examples in section 4 and in [1]. Other efficient solvers for boundary value problems (on large intervals) could be adapted for numerical solutions of (14); we shall not address this any further here.

2.4. Systems with continuous symmetries. It can often happen that the system (1) commutes with the action of a $k$-dimensional compact Lie group $\Gamma$. Let $\Gamma_{0}$ be the connected component of the identity in $\Gamma$. A relative equilibrium is defined to be a dynamically invariant group orbit of a single point.

Assume that we have a trajectory $q(t)$ which limits to a relative equilibrium $p$ both forwards and backwards in time. Assume that the endpoints and the connection have trivial isotropy and so in particular, for each finite value of $t, \Gamma_{0} q(t)$ is a $k$ dimensional manifold. We cover a neighborhood of $q(t)$ by a global product $\Gamma_{0} \times X$ where $X$ is a domain in a linear subspace of $R^{n}$ (this may cover the endpoints twice). We investigate the relative homoclinic orbit by lifting it to $X$; in this subspace it is a connecting orbit. Note that the existence of a group action can cause there to be such orbits in a structurally stable manner (see [12] and the example in section 4.2). This is typically due to the fact that the group action leaves invariant subspaces where the connection is from a saddle (relative equilibrium) to a sink.

The linear variational problem of (1) is then

$$
\frac{d z}{d t}=D_{x} f(q(t), 0) z .
$$

In the least degenerate case this has a $k+1$-dimensional solution space, i.e.,

$$
\operatorname{ker}\left(\frac{d}{d t}-D_{x} f(q(t), 0)\right)=\operatorname{span}\left[z_{1}, z_{2}, \ldots, z_{k+1}\right] .
$$

We can set $z_{k+1}(t)=c \dot{q}(t), c \in R$ and $z_{i}(t)=\gamma_{i} q(t), i=2, \ldots, k+1$, where $\left\{\gamma_{i}\right\}$ generates the Lie algebra of $\Gamma$. In other words, once the homoclinic orbit $q(t)$ is known, we derive $z_{k+1}$ by differentiation with respect to $t$ and $z_{i}, i=1, \ldots, k$ by the infinitesimal group action. Similarly, the kernel

$$
\operatorname{ker}\left(\frac{d}{d t}+D_{x} f(q(t), 0)^{T}\right)=\operatorname{span}\left[\psi_{1}, \psi_{2}, \ldots, \psi_{k+1}\right]
$$

is also $k+1$ dimensional. The equality

$$
\langle\psi(t), z(t)\rangle=\text { constant }
$$

holds for a given solution $z(t)$ of the adjoint problem. However, because $p$ is nonhyperbolic the constant need not vanish, i.e., $\psi_{i}$ and $z_{i}$ do not necessarily decay at 
infinity. For this reason we can calculate a basis $\left\{\psi_{i}\right\}$ from the following system:

$$
\begin{aligned}
\left\langle\psi_{i}, z_{j}\right\rangle & =\delta_{i j}, \quad(i, j)=1, \ldots, k, \\
\left\langle\psi_{i}, z_{k+1}\right\rangle & =0, \quad i=1, \ldots, k+1, \\
\left(I-\sum_{j=1}^{k+1} z_{j} z_{j}^{T}\right)\left[\frac{\partial}{\partial t}+\left(D_{x} f(q(t), 0)\right)^{T}\right] \psi_{i} & =0, \\
\tilde{L}_{s}^{*} \psi_{i}(T) & =0, \\
\tilde{L}_{u}^{*} \psi_{i}(-T) & =0, \\
\Phi\left(\psi_{i}\right) & =0 .
\end{aligned}
$$

The unique decaying bounded solution of this system will be $\psi_{k+1}$. A shooting method approximation of this system (cf. (15)) is used by us in [1].

3. The approximate bifurcation function. Given $q(t)$ and $\psi(t)$, it is possible to obtain the leading terms of the bifurcation function by [15, Eq. (4.7)] as

$$
\begin{aligned}
G(\omega, \mu) & =\psi(\omega) q(-\omega)-\psi(-\omega) q(\omega)+\mu \int_{-\infty}^{\infty} \psi(s) D_{\mu} f(q(s), 0) d s \\
& +o\left(|\mu|+e^{-\delta \omega}\right)
\end{aligned}
$$

for $\omega \in[0, T]$, where $\delta>2 \rho$ and $\rho$ is a real part of the leading eigenvalue $\rho \pm i \beta$ of $D_{x} f$ at the fixed point. In particular, for generic situations we have [15, Thm. 4.6]

$$
G(\omega, 0)=e^{-2 \rho \omega} \Theta(\omega)+\text { h.o.t., }
$$

where $\Theta$ is a quasiperiodic function of $\omega$, a sum of trigonometric functions of period $\pi / \beta$, and h.o.t. are higher-order terms that decay at an exponentially faster rate.

We can fit $q(t)$ and $\psi(t)$ obtained by numerical approximation at the points $t= \pm T$ in the following way: Suppose that $\left\{\lambda_{i}, v_{i}\right\}$ are the leading eigenpairs ${ }^{1}$ of $D_{x} f(0,0)$ and $\operatorname{Re}\left(\lambda_{i}\right)=\rho>0$. Similarly, let $\left\{\lambda_{i}, v_{i}^{*}\right\}$ be the leading eigenpairs of $\left(D_{x} f(p, 0)\right)^{T}$. We make the generic assumption that $D_{x} f(p, 0)$ is semisimple, i.e., complex diagonalizable. There is a $\delta>0$ and real quantities $A_{i}, B_{i}$ such that

$$
q(t)=\sum_{i} A_{i} v_{i} e^{\lambda_{i} t}+o\left(e^{(\rho+\delta) t}\right)
$$

for $t \rightarrow-\infty$, whereas for $t \rightarrow \infty$ we have

$$
\psi(t)=\sum_{i} B_{i} v_{i}^{*} e^{-\lambda_{i} t}+o\left(e^{-(\rho+\delta) t}\right) .
$$

We can approximate the coefficients $A_{i}$ and $B_{i}$ by fixing $t$ to be one of the endpoints, $\pm T$, taking inner products of these equations with $\left\{v_{j}^{*}\right\}$, resp., $\left\{v_{j}\right\}$ and then solving the resulting linear system for $A_{i}, B_{i}$.

In this way we can obtain the leading order behavior of $G(\omega, 0)$. In the case described above (which includes both examples discussed in section 4) we write

$$
\begin{aligned}
G(\omega, 0) & =\psi(\omega) q(-\omega)-\psi(-\omega) q(\omega)+\text { h.o.t. } \\
& =-\psi(-\omega) q(\omega)+\text { h.o.t. } \\
& =-\sum_{i, j} A_{i} B_{j} e^{-\left(\lambda_{i}+\lambda_{j}\right) \omega}\left\langle v_{j}^{*}, v_{i}\right\rangle+\text { h.o.t. }
\end{aligned}
$$

\footnotetext{
${ }^{1}$ That is, corresponding to eigenvalues $\lambda_{i}$ of $D_{x} f(p, 0)$ that are closest to the imaginary axis.
} 
The integral term in (4) can be approximated by numerical integration, e.g., by Simpson's rule. Thereafter, we obtain an approximate bifurcation function and can extract information about a continuum of periodic orbits with asymptotically large periods. One can see this as analogous to the prediction of branches of stationary solutions near a steady-state bifurcation point with the reduced bifurcation equations in the Liapunov-Schmidt reduction.

Since it is necessary to compute $q(t)$ and $\psi(t)$ for detection of degenerate homoclinic orbits in continuation problems [5], we remark that one can potentially get a lot more information from such continuations at little extra computational cost.

Continuation of homoclinic orbits with respect to parameters. Once the adjoint variational problem is solved, we are in a good position to make a predictor for the purpose of continuation of homoclinic orbits. Note that for multiparameter problems the hyperplane

$$
\left\{\nu \in R^{d}: \sum_{i} \nu_{i} \int_{-\infty}^{\infty}\left\langle\psi(t), \frac{\partial f}{\partial \nu_{i}}(q(t), 0)\right\rangle d t=0\right\}
$$

corresponds to all perturbations $\nu$ in parameter space $R^{d}$ for which the variational problem

$$
\dot{u}(t)-D_{x} f(q(t), 0) u(t)=D_{\mu} f(q(t), 0) \nu ;
$$

i.e., the linearization of (1) with respect to $\mu$ at $(q(t), 0)$, has bounded solution. Moreover, the linear operator $\frac{d}{d t}-D_{x} f(q(t), 0)$ has a one-dimensional kernel $\operatorname{span}[\dot{q}(t)]$ and we are at liberty to select solutions of this equation that satisfy

$$
\langle\psi(t), u(t)\rangle=0
$$

In other words, one can interpret the "Melnikov" integral as a solvability condition. For one-parameter problems the nondegeneracy condition (7) implies that the homoclinic orbit is isolated.

Given a $\nu$ and a solution $u(t)$ of the above equation, one thus obtains a linear predictor

$$
(q(t), 0) \mapsto(q(t)+\epsilon u(t), \epsilon \nu)
$$

for continuation of the homoclinic orbit.

\section{Examples.}

4.1. Freire, Rodriguez-Luis, and Ponce's circuit. As an example of ODEs with interesting periodic orbit structure near a homoclinic orbit, we consider the following equations of Freire, Rodriguez-Luis, and Ponce [9], also used as a test system by Champneys and Kuznetsov [4]:

$$
\begin{aligned}
r \dot{x} & =-\nu x+\beta(y-x)-A_{3} x^{3}+B_{3}(y-x)^{3}, \\
\dot{y} & =-\beta(y-x)-z-B_{3}(y-x)^{3}, \\
\dot{z} & =y,
\end{aligned}
$$

where $(x, y, z) \in R^{3}, r=0.6, A_{3}=0.328578, B_{3}=0.933578$. We consider $(\nu, \beta)$ as free parameters. This system has a fixed point at the origin, and we find an approximate homoclinic orbit and solution to the adjoint variational equation as described in 
section 2. This allows us to compute the approximate bifurcation function $G(\omega, \mu)$ as in section 3. Approximations of the homoclinic orbit and the solution of the AVE are shown in Figure 1. This was done by a collocation in the time interval $[-17,17]$ for the solution of the homoclinic orbit and Gear's method shooting with the projection correction for the solution of the AVE. Note that $\psi$ is shown at the gridpoints with linear interpolation; in fact, the computed solution has higher accuracy than might appear to be the case.

The corresponding bifurcation function and a comparison with a bifurcation diagram computed by AUTO86 [8] is shown in Figure 2. The curve labelled "AUTO" is the path-followed curve, and the prediction from truncating (4) is labelled " $G=0$ prediction." The other curve is obtained using an exponential fit and (18). As can be seen, all three curves are in excellent agreement, even right down to near the Hopf bifurcation at $(\nu, \beta)=(0.55,0.3)$. As expected, the exponential fit has higher errors and moves away from the true curve before the " $G=0$ prediction" curve does, but has the advantage that it provides an extrapolation of the bifurcation function to periods longer than the interval in which $q(t)$ is calculated. Figure 3 shows the error of the leading exponential fit to the AUTO prediction of the value of $\nu$ necessary for the appearance of a periodic orbit of a given period. Note that the error decays exponentially fast up to approximately $\omega=40$, where it levels off at the error tolerance used for the AUTO calculation.

4.2. Kuramoto-Sivashinsky equation. As a second example, this time for a discretization of a parabolic PDE with the symmetry of a continuous group, we consider what additional information this method can give us to the problem studied in [1].

In that paper we considered the perturbed Kuramoto-Sivashinsky equation on the interval $[0,2 \pi)$ defined by

$$
\begin{aligned}
0 & =\frac{\partial v}{\partial t}-F(v ; \alpha, \gamma) \\
& :=\frac{\partial v}{\partial t}+4 v_{x x x x}+\alpha\left(v_{x x}+v v_{x}\right)-\gamma v_{x x x}
\end{aligned}
$$

where $\alpha, \gamma \in R$ are parameters and $v(x, t)$ satisfies periodic boundary conditions

$$
v(0, t)=v(2 \pi, t)
$$

and an initial value

$$
v(x, 0)=v_{0}
$$

The parameter $\gamma$ can be used to break the $x$-reflection symmetry. If $\gamma=0$ and $\alpha \in(16.1399,22.557)$ there are attracting structurally stable homoclinic orbits (see, e.g., [12]). These homoclinic cycles bifurcate to two-frequency quasiperiodic attractors for $\gamma \neq 0$ (see, e.g., [6] and [1]).

In [1] we concentrated on the problem of calculating the integral term in (4); we note here that we were easily able to modify the program to also approximate $G(\omega, 0)$ in the limit $\omega \rightarrow \infty$. We use the notation and $\alpha=22$ as in [1]. This gives a homoclinic orbit $q(t)$ between two fixed points $u^{ \pm}$on the same group orbit such that

$$
q( \pm t) \rightarrow u^{ \pm} \quad \text { as } \quad t \rightarrow \infty
$$




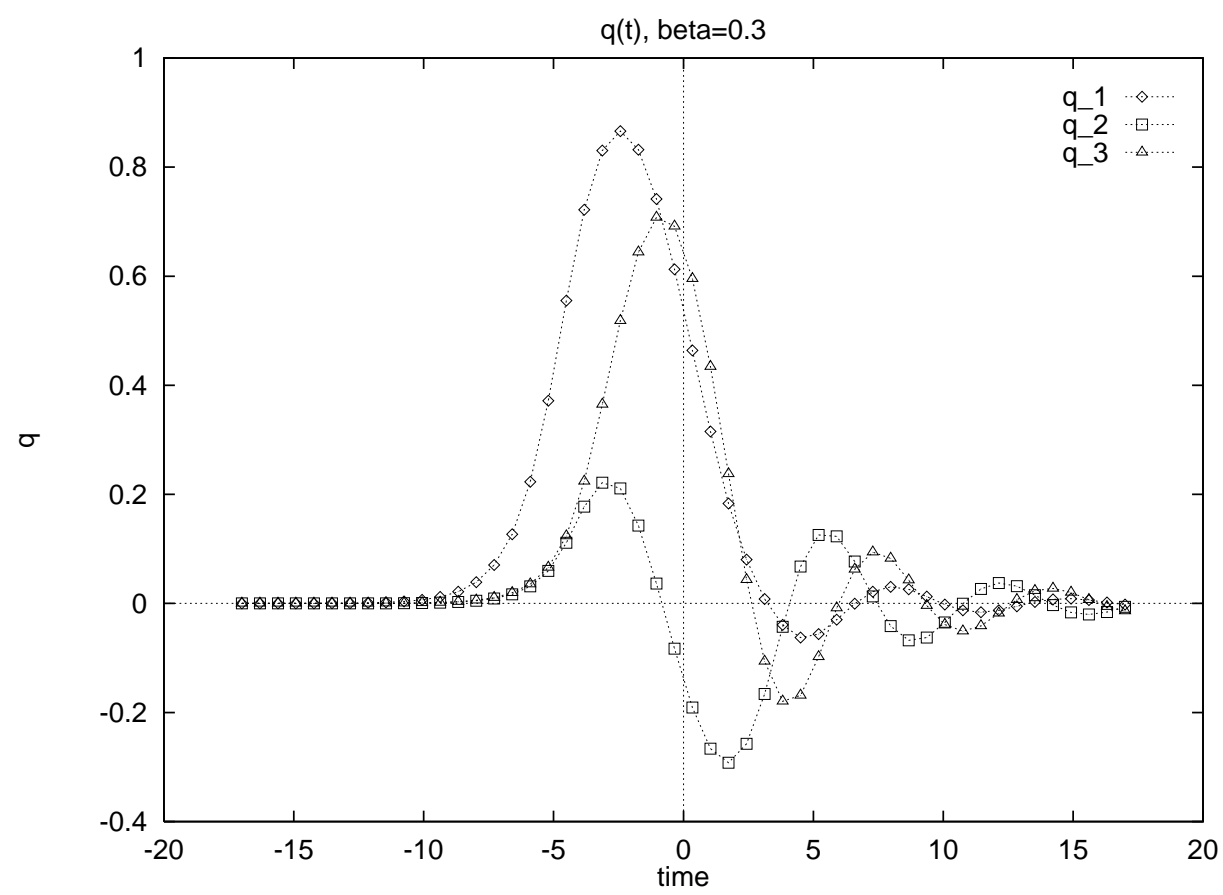

(i)

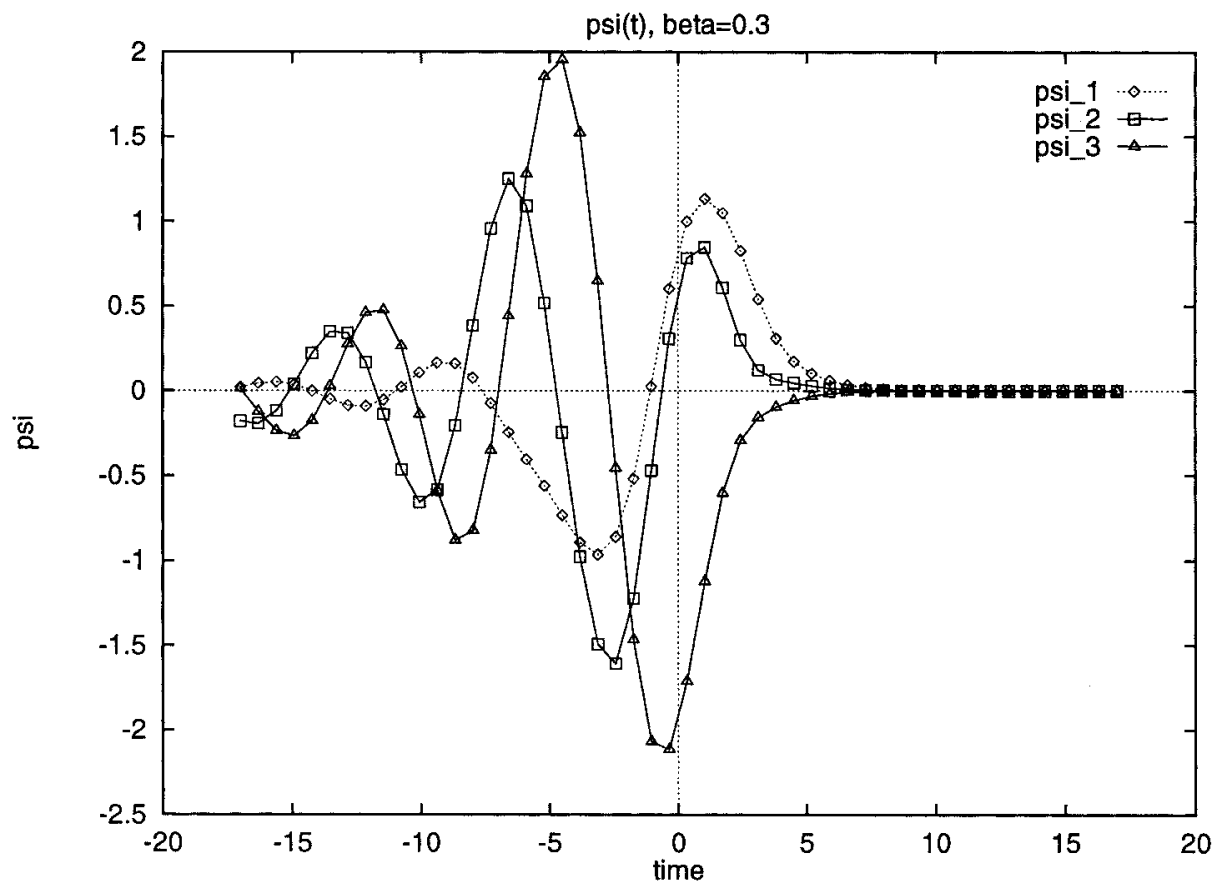

(ii)

FIG. 1. Approximations of the three components of the homoclinic orbit $q(t)$ and the solution of the adjoint variational equations $\psi(t)$ for Freire, Rodriguez-Luis, and Ponce's circuit equations, on the truncated time interval $[-17,17]$. Note that the plotted points are calculated to higher accuracy than the piecewise linear interpolation suggests. The homoclinic orbit is located at $(\nu, \beta)=(-0.775883,0.3)$. 


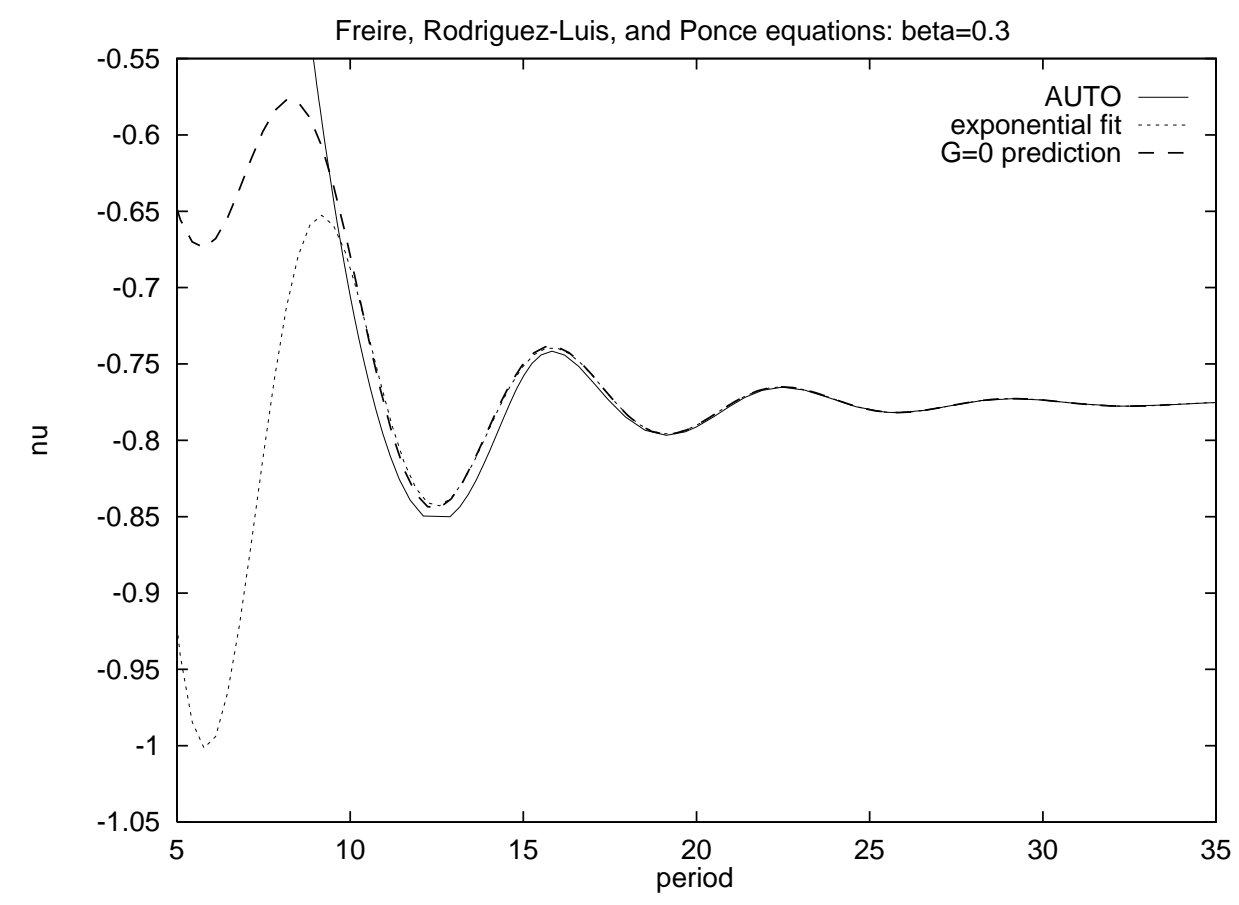

FIG. 2. Computed bifurcation diagram at $\beta=0.3$ in the $(P, \nu)$ plane $(P=2 \omega)$. The three curves correspond to a path followed curve from AUTO86; the approximate zeros of the bifurcation function with $G(\omega, 0)$ calculated by exponential fit and by direct numerical calculations, respectively. Note that all three curves remain close right down to a neighborhood of the Hopf bifurcation at $\nu=-0.55$ that creates the periodic orbit.

The single unstable eigenvalue is $\lambda=8.269$, while the leading stable eigenvalue is -50.88 (there is also a neutral eigenvalue given by the continuous group action). The eigenvector of the linearization at $u^{ \pm}$corresponding to $\lambda$ we refer to as $e_{u}^{ \pm}$while that of the adjoint is $e_{u}^{* \pm}$. The homoclinic orbit and solution of the AVE were calculated on the time-interval $[-1.5,1.5]$. The leading terms in $G(\omega, 0)$ are of the form

$$
G(\omega, 0)=C e^{-2 \lambda \omega}+I \gamma+\text { h.o.t. }
$$

where the constant $C$ is calculated from fitting exponentials to the endpoints as described in section 3. The integral term $I$ is calculated as in [1]. Table 1 shows $C$ and $I$ calculated as functions of a spatial discretization using $2 N$ Galerkin modes and maximum stepsize $\delta$ for shooting with the Gear's method. Thus we get the approximation

$$
G(\omega, \gamma)=0.3231 \exp (-16.538 \omega)+0.475 \gamma+o(\gamma+\exp (-16.538 \omega))
$$

The signs of the constants imply that we should see quasi-periodicity in the original equations for small $\gamma<0$ (see [1] for more details) that remains close to the group orbit of the original homoclinic cycle. Figure 4 shows good agreement between measured period from direct simulation of 16 mode truncated equations (calculated using a variable-step Runge-Kutta integrator) and the prediction from (20). One also gets quasi-periodicity for small $\gamma>0$; this is seen by considering the symmetry $(x, \gamma) \rightarrow$ $(2 \pi-x,-\gamma)$. 


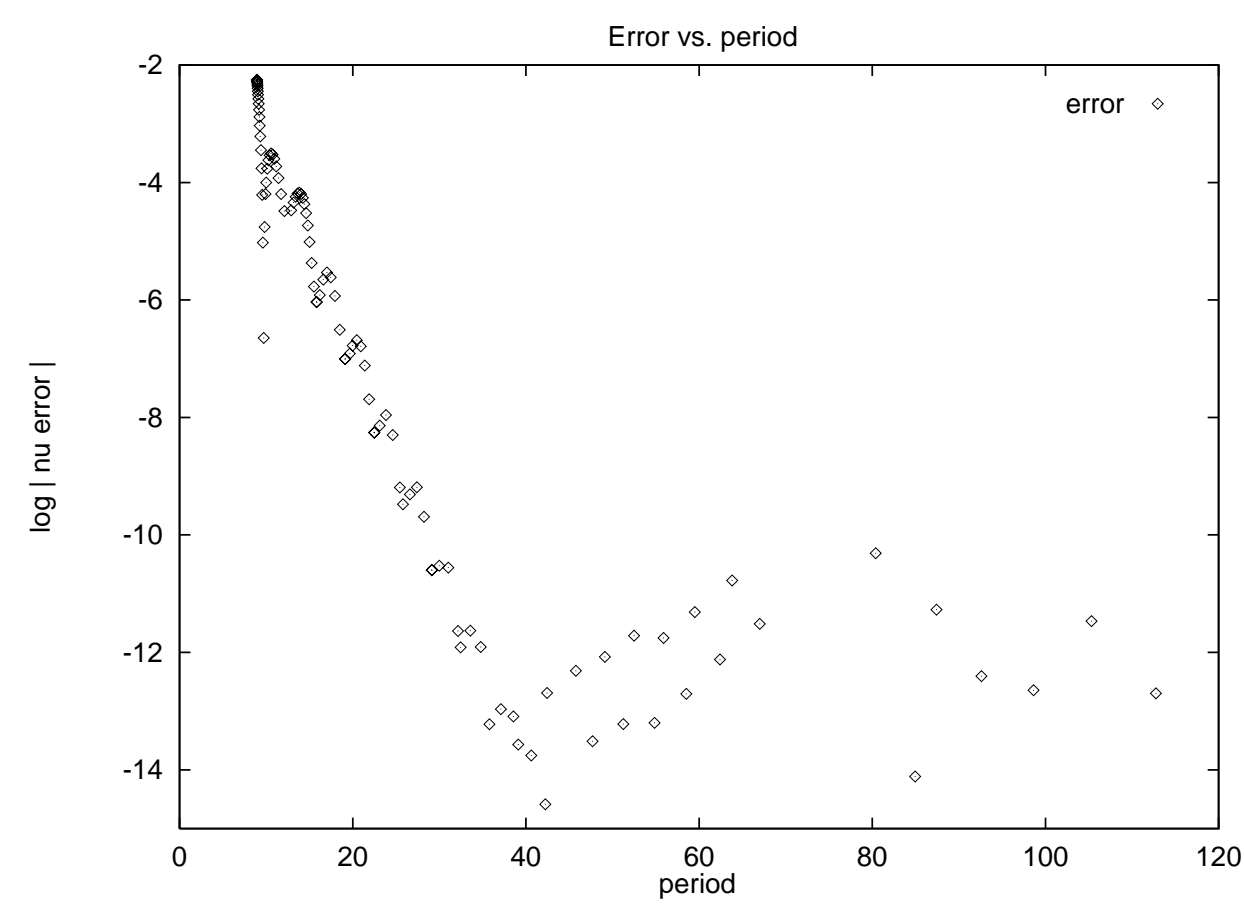

FIG. 3. Log of the absolute difference between the values $\nu$ for a periodic orbit of a given period calculated by using an exponential fit to the bifurcation function and by AUTO86. Note the exponential convergence of the methods with periods up to 40. After that the error saturates at an error smaller than $e^{-10}$. The error decays much faster than the rate of convergence of the parameter to the homoclinic orbit (this is evident in Figure 2).

TABLE 1

Constants for the approximation of the bifurcation function for the perturbed KuramotoSivashinsky equation. $N$ and $\delta$ are the spatial and temporal discretization parameters, respectively.

\begin{tabular}{cc|cc}
$N$ & $\delta$ & $C$ & $I$ \\
\hline 8 & 0.4 & -0.3054 & 0.4698 \\
8 & 0.2 & -0.3231 & 0.4747 \\
16 & 0.2 & -0.3228 & 0.4747 \\
16 & 0.1 & -0.3308 & 0.4753 \\
\hline
\end{tabular}

5. Conclusions. Methods for numerical analysis of bifurcation problems usually fall into one of three categories - continuation of solution branches, detection of bifurcation points, and analysis of bifurcations for branch switching (see, e.g., Seydel [18] and Kuznetsov [13, Chapter 10]. The main contribution of what we have discussed here for the numerical analysis of dynamics near a homoclinic orbit is a branch switching method for finding less degenerate solutions (periodic orbits) near a more degenerate solution (a homoclinic orbit). This involves approximating a numerical bifurcation function from Lin's method [15] and determining the relations between the parameters and the periods of periodic solutions, which can be used as an accurate predictor of periodic solutions near a homoclinic orbit.

This bifurcation function can be constructed from data that is already available if one wants to detect bifurcations associated with codimension two homoclinic orbits. 


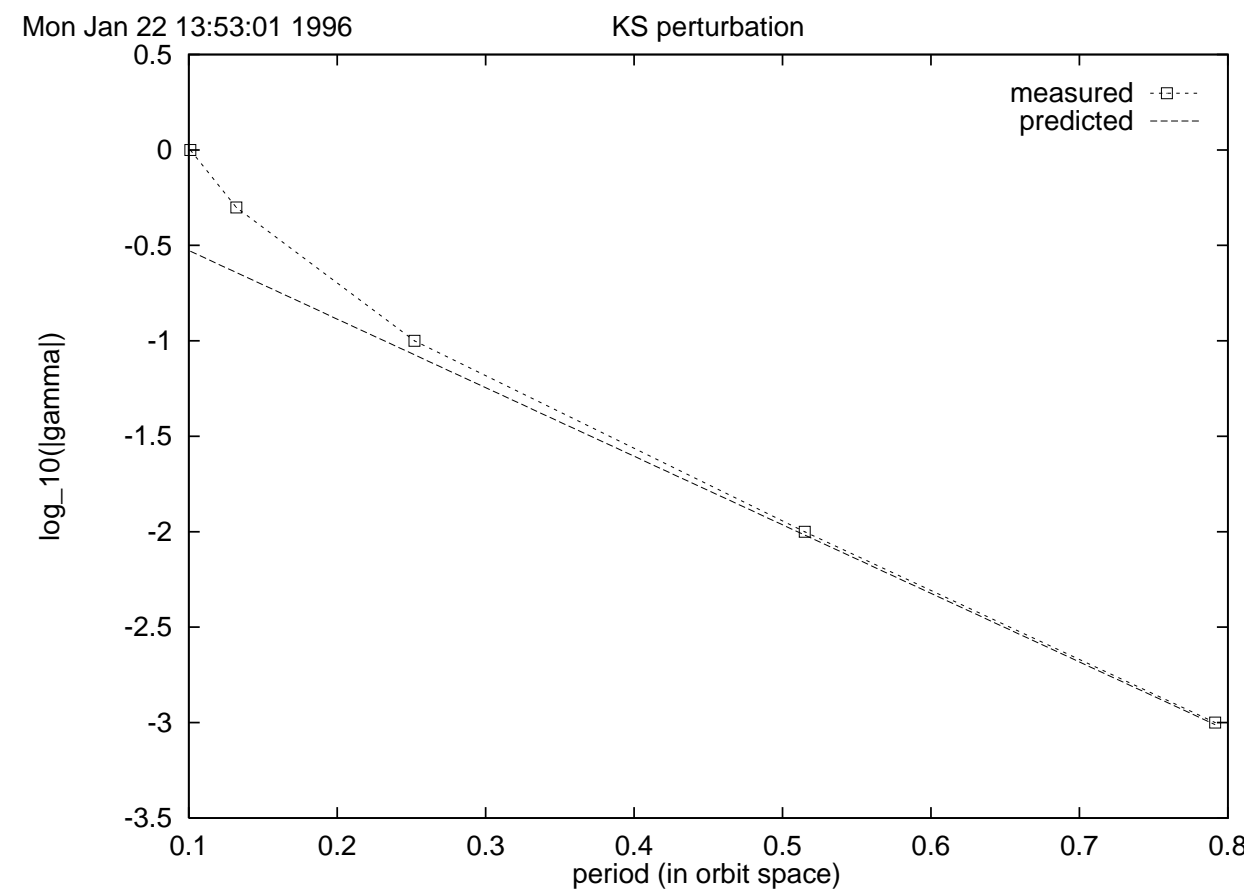

FIG. 4. Periods of (relative) periodic solutions in the orbit space with respect to the $O(2)$ symmetry for the perturbed Kuramoto-Sivashinsky equation as a function of $\log _{10}(|\gamma|)$. The points indicate measurements from simulations of 16 mode truncated equations. The prediction is from (20).

In addition to providing information about nearby periodic orbits, we easily obtain a linear predictor for continuation of homoclinic orbits.

We have also introduced a class of methods for solving the adjoint variational equation while preserving the inner product; however, the numerical bifurcation function should be constructible using other sensible methods for solving this variational equation.

The two numerical examples show good agreement of this method with direct calculations of the branch of periodic solutions. Another approach to obtain $G(\omega, \mu)$ for moderately small $\omega$ would be to numerically solve the boundary value problem (3) directly; it is envisaged that knowledge of $\psi$ may provide good starting approximations for this problem.

Acknowledgments. We thank Björn Sandstede and the referees for some very helpful comments on this paper.

\section{REFERENCES}

[1] P. Ashwin, K. Böhmer, And Z. MeI, Forced symmetry breaking of homoclinic cycles in a PDE with $O(2)$ symmetry, J. Comput. Appl. Math., 70 (1996), pp. 297-310.

[2] W.-J. BEYN, Global bifurcations and their numerical computation, in Continuation and Bifurcations: Numerical Techniques and Applications, D. Roose et al., eds., Kluwer Academic Publishers, Norwell, MA, 1990, pp. 169-181.

[3] W.-J. BEYN, The numerical computation of connecting orbits in dynamical systems, IMA J. Numer. Anal., 9 (1990), pp. 379-405. 
[4] A.R. Champneys and Yu.A. Kuznetsov, Numerical detection and continuation of codimension-two homoclinic bifurcations, Internat. J. Bifur. Chaos Appl. Sci. Engrg., 4 (1994), pp. $795-822$.

[5] A.R. Champneys, Yu.A. Kuznetsov, and B. Sandstede, A Numerical Toolbox for Homoclinic Bifurcation Analysis, University of Bristol, Department of Engineering Maths., Bristol, England, Report 1.95, 1995.

[6] P. Chossat, Forced symmetry breaking of an $\mathrm{O}(2)$-symmetric homoclinic cycle, Nonlinearity, 6 (1993), pp. 723-731.

[7] E. Doedel, AUTO: A program for the automatic bifurcation analysis of autonomous systems, Congr. Numer., 30 (1981), pp. 265-284.

[8] E. Doedel And J.P. Kernevez, AUTO: Software for Continuation and Bifurcation Problems in Ordinary Differential Equations, Tech. report, California Institute of Technology, Pasadena, CA, 1986.

[9] E. Freire, A. Rodriguez-Luis, and E. Ponce, A case study for homoclinic chaos in an autonomous electronic circuit: A trip from Takens-Bogdanov to Hopf-Shilnikov, Physica D, 62 (1993), pp. 230-253.

[10] B. HASsard, Computation of invariant manifolds, in New approaches to Nonlinear Problems, P. Holmes, ed., SIAM, Philadelphia, 1980, pp. 27-42.

[11] F.R. DE HoOg AND R. WeISs, An approximation theory for boundary value problems on infinite intervals, Computing, 24 (1980), pp. 227-239.

[12] I.G. Kevrekidis, B. Nicolaenko, and J.C. Scovel, Back in the saddle again: A computer assisted study of the Kuramoto-Sivashinsky equation, SIAM J. Appl. Math., 50 (1990), pp. $760-790$.

[13] Y. A. Kuznetsov, Elements of Applied Bifurcation Theory, Springer-Verlag, New York, 1995.

[14] M. Lentini And H.B. Keller, Boundary value problems on semi-infinite intervals and their numerical solution, SIAM J. Numer. Anal., 17 (1980), pp. 577-604.

[15] X.-B. Lin, Using Melnikov's method to solve Silnikov's problems, Proc. Roy. Soc. Edinburgh, 116A (1990), pp. 295-325.

[16] G. Moore, Computation and parametrization of periodic and connecting orbits, IMA J. Numer. Anal., 15 (1995), pp. 245-263.

[17] B. Sandstede, Private communication, 1995.

[18] R. SEydel, From Equilibrium to Chaos-Practical Bifurcation and Stability Analysis, Elsevier, New York, Amsterdam, London, 1988.

[19] A. Vanderbauwhede And B. Fiedler, Homoclinic period blow-up in reversible and conservative systems, Z. Angew. Math. Phys., 43 (1992), pp. 292-318. 